\title{
Effects of treatment with low molecular weight phenol formaldehyde resin on the surface characteristics of oil palm (Elaeis quineensis) stem veneer.
}

\begin{abstract}
Even though oil palm (Elaeis quineensis) stem (OPS) is highly potential as an alternative raw material in wood industry, it possesses some inferior characteristics. One of the critical weaknesses is a high degree of veneer surface roughness that resulted in high resin consumption during plywood manufacture. The objective of this study was to investigate effects of treatment with low molecular weight phenol formaldehyde (LMWPF) resin on the wettability and surface roughness of OPS veneer. OPS veneers were segregated into two categories namely outer and inner layer veneer, prior to soaking in LMWPF resin solution to obtain weight percent gain of 16-20\%. The wettability of OPS veneers was assessed with contact angle measurement according to the sessile drop method. The veneer surface roughness was evaluated by determining the average roughness (Ra), mean peak-to-valley height $(\mathrm{Rz})$, and maximum roughness (Rmax) using a stylus profilometer in accordance with DIN standard 4768. The results show that the effect of LMWPF resin treatment on the surface roughness of the veneers is statistically significant. The technique used in the study was able to enhance the surface properties as well as improved the physical and mechanical properties of OPS plywood.
\end{abstract}

Keyword: Natural materials; Surface treatments; Mechanical. 